\title{
Individualisierte (personalisierte) Therapie - jetzt auch in der Rehabilitation?
}

Der Rehabilitationsbedarf wird in den nächsten Jahren ständig steigen - darin sind sich alle einig. Demographische Entwicklungen (Zunahme der rehabilitationsintensiven Altersgruppen), höheres Renteneintrittsalter, gestiegene Anforderungen in der Arbeitswelt sowie sich frühzeitig im Erwerbsleben manifestierende Erkrankungen steigern die Nachfrage nach qualitativ hochwertigen Reha-Angeboten.

In Deutschland hat sich in den letzten beiden Jahrzehnten eine hochspezialisierte Rehabilitationsmedizin entwickelt, die sich heute neben Prävention und Akuttherapie als wichtige Säule des Gesundheitssystems präsentiert. Der größte Anteil der Rehabilitationsmaßnahmen wird von der Deutschen Rentenversicherung finanziert, die die Kosten für Rehabilitationen von erwerbstätigen Patienten aller Indikationen sowie in der Onkologie zusätzlich auch von Rentnern und mitversicherten Angehörigen (nach §31 SGB VI) übernimmt. Sicher auch unter dem (Ein)druck der Rehakrise im Jahr 1996 wurden bundesweit flächendeckende Qualitätssicherungsprogramme eingeführt, weiterentwickelt sowie umfangreiche Rehabilitationsforschungsprogramme etabliert. Das Image hat sich von der klassischen Kur zur modernen Rehabilitation deutlich gewandelt; das bio-psycho-soziale Krankheitsmodell der WHO und die funktionale Gesundheit bilden die Grundlage der Rehabilitation. Demzufolge versteht sich erfolgreiche Rehabilitation als das Ergebnis interdisziplinärer Arbeit, für deren Erfolg neben Ärzten Psychologen, Sport- und Bewegungstherapeuten, Sozialpädagogen, Ernährungstherapeuten u.a. unabdingbar sind.

Rehabilitiert werden überwiegend Menschen mit chronischen Krankheiten, oft Zivilisationskrankheiten, die häufig auch durch Umweltfaktoren (Ernährung, mangelnde Bewegung, Stress) bedingt sind. Damit steht die Lebensstilmodifikation seit jeher im Zentrum aller therapeutischen Bemühungen der Rehabilitation. Langfristige Änderungen des Lebensstils sind in der Realität nur sehr schwer und von wenigen Patienten umsetzbar. Viele gute Ansätze in der Rehabilitation sind schwierig im Alltag zu integrieren. Dies umso mehr, als bislang die Sektorengrenzen zwischen Prävention, Akutmedizin und Rehabilitation kaum durchlässig sind und die beteiligten Akteure sich allzu oft kaum kennen bzw. über aktuelle Therapiekonzepte des jeweiligen Gegenübers kaum informiert sind.

Daher freut es mich besonders, dass die DMW in dieser Ausgabe ein rehabilitationsmedizinisches Thema aufgreift. Individualisierte (personalisierte) Therapie ist derzeit ein „Mega-Trend“ in der Akut-
Onkologie. Gilt dies auch für die RehabilitationsOnkologie? Ökonomische Erwägungen und die Einführung von Qualitätssicherungsmaßnahmen haben in den letzten Jahren eine zunehmende Standardisierung von Reha-Angeboten zur Folge gehabt. Dabei haben wir es in der Onkologie mit einem äußerst inhomogenen Patientenklientel zu tun. Alter, Geschlecht, Diagnosen, Krankheitsstadium, kurative oder palliative Therapie, Begleitkrankheiten etc. sind Faktoren, die bei den Rehabilitationsinhalten berücksichtigt werden müssen.

Stehen eher psychosoziale Folgen im Fokus der Therapie oder geht es darum, die körperliche Aktivität anhaltend zu steigern, um Begleiterkrankungen und die eigenen Krankheitsprognose positiv zu beeinflussen? Kann überhaupt innerhalb von nur 3 Wochen (derzeitige Regeldauer bei onkologischen Rehabilitationen 22 Tage) eine dauerhafte Lebensstilmodifikation erreicht werden? Müssen Therapiekonzepte und -inhalte nicht auch in der onkologischen Rehabilitation individualisiert werden, um eine Unter- und Übertherapie zu vermeiden? Wie lässt sich das Rehabilitationsergebnis optimieren? Könnte auch eine zunehmende Flexibilisierung der Reha-Dauer, z.B. durch stationär-ambulante Kombi-Angebote, Etappen-Wochen, Telefon-Coaching, für den langfristigen Erfolg einer Maßnahme von Bedeutung sein? Bedeutet individualisierte Rehabilitation nicht auch, dass wir den vor- und nachbehandelnden Arzt, d.h. unterschiedliche Disziplinen über Sektorengrenzen hinweg einbinden müssen, damit Therapien im Sinne und zum Wohl des Patienten langfristig fortgeführt werden können? Viele Fragen, die wir klären müssen, um Rehabilitation zukunftsfähig zu machen. Wie ein solch individualisiertes Konzept zu einer dauerhaften Steigerung der körperlichen Aktivität bei Brustkrebspatientinnen beispielhaft umgesetzt werden kann, stellen wir im Artikel ab S. 895 ausführlicher dar.

„Rehabilitation 2013 - Erfolg durch Interdisziplinarität" lautet das Motto des diesjährigen Kongresses der Rehazentren Baden-Württemberg, der vom 2.4.5.2013 in Stuttgart stattfindet. Ich würde mich freuen, wenn Ihr Interesse an moderner Rehabilitationsmedizin mit dem Artikel geweckt wäre. Und vielleicht sehen wir uns ja auf der Tagung, um mit Ihnen persönlich und allen Beteiligten über neue interdisziplinäre Konzepte konstruktiv und durchaus auch kontrovers zu diskutieren.

\section{Ihre}

Prof. Dr. Monika Reuss-Borst

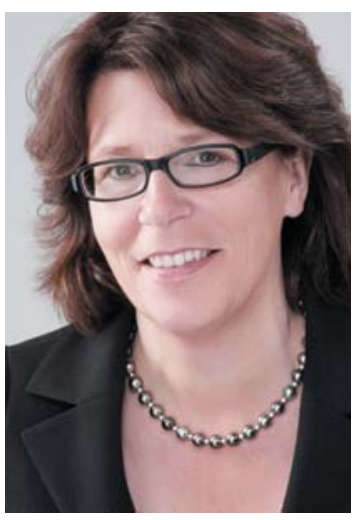

M. A. Reuss-Borst

Rehabilitation

Institut

Rehazentren

Baden-Württemberg,

Stuttgart

Bibliografie

Dol $10.1055 / \mathrm{s}-0033-134312$

Dtsch Med Wochenschr 2013;

138: 879 - (c) Georg Thieme Verlag KG · Stuttgart · New York · ISSN 0012-0472

Korrespondenz

Prof. Dr. Monika Reuss-Borst

Klinik am Kurpark, Rehazentren Baden-Württemberg Kurhausstr. 9

97688 Bad Kissingen eMailm.reuss-borst@ rehazentren-bw.de 